\title{
VARIAÇÃO DOS NÍVEIS DE HEMOGLOBINA DE PACIENTES EM HEMODIÁLISE TRATADOS COM ERITROPOETINA: UMA EXPERIÊNCIA BRASILEIRA
}

\author{
Adriano Luiz Ammirati', Renato Watanabe ${ }^{2}$, Cristiane Aoqui ${ }^{3}$, Sergio Antonio Draibe 4 , Aloisio Barbosa Carvalho 5 , Hugo Abensur 6 , Soraia Stael Drumond ${ }^{7}$ \\ João Moreira ${ }^{8}$, José Luis Bevilacqua ${ }^{9}$, Anita CM Silva ${ }^{10}$, Fernando Tatsch ${ }^{11}$, Maria Eugenia Canziani ${ }^{12 *}$
}

Trabalho realizado na Universidade Federal de São Paulo - UNIFESP, São Paulo, SP

\section{*Correspondência:}

Rua Pedro de Toledo, 282

São Paulo - SP

CEP: 04039-000

Telefone: (11) 5904-8499 -

Fax: (11) 5572-1862

dialisefor@uol.com.br

\begin{abstract}
RESUMO
Овлетіvo. Avaliar a variabilidade dos níveis de hemoglobina ( $\mathrm{Hb}$ ) em pacientes em hemodiálise (HD) tratados com eritropoetina.

MÉTodos. Foram coletados dados retrospectivos de 249 pacientes que estavam em HD e apresentavam, nos três meses anteriores, média de $\mathrm{Hb}$ entre $10,5 \mathrm{~g} / \mathrm{dL}$ e $12,5 \mathrm{~g} / \mathrm{dL}$. O período de observação total foi de 36 meses. A cada mês de coleta, classificaram-se os valores de $\mathrm{Hb}$ em: $<10,5 \mathrm{~g} / \mathrm{dL}, 10,5 \mathrm{~g} /$ $\mathrm{dL}<\mathrm{Hb}<12,5 \mathrm{~g} / \mathrm{dL}$ (intervalo alvo), ou $\mathrm{Hb}>12,5 \mathrm{~g} / \mathrm{dL}$. Além disto, os pacientes foram divididos em seis categorias de variabilidade da $\mathrm{Hb}$ : baixo persistente $(<10,5 \mathrm{~g} / \mathrm{dL})$, alvo persistente $(10,5$ a 12,5 $\mathrm{g} / \mathrm{dL}$ ), alto persistente ( $>12,5 \mathrm{~g} / \mathrm{dL}$ ), flutuação de baixa amplitude com Hb baixo, flutuações de baixa amplitude com $\mathrm{Hb}$ alto e flutuações de alta amplitude.

Resultados. Mês a mês, a média da proporção de pacientes com Hb dentro da faixa alvo foi de $50 \%$ (variação, $42 \%$ a $61 \%$ ). A proporção de valores de $\mathrm{Hb}$ médios acima da faixa alvo (30\%) foi mais frequente que a proporção abaixo do alvo (20\%). Durante os períodos de seis, 12, e 36 meses, a proporção de pacientes com $\mathrm{Hb}$ baixa persistente se reduziu de $3,6 \%$ para $0 \%$; de $31,7 \%$ para $2,8 \%$ naqueles com $\mathrm{Hb}$ alta persistente; de 7,6\% para $0 \%$ naqueles com baixa amplitude com $\mathrm{Hb}$ baixo; e de $41,3 \%$ para $8,3 \%$ nos pacientes com baixa amplitude com $\mathrm{Hb}$ alto. Entretanto, houve aumento na proporção de pacientes (de $21,5 \%$ a $88,9 \%$ ) com alta amplitude de $\mathrm{Hb}$. Portanto, à medida que 0 tempo de observação se alongou observou-se maior variabilidade dos valores de hemoglobina. Nenhum paciente manteve os níveis de $\mathrm{Hb}$ dentro do alvo durante todo o período do estudo.

Conclusão. A manutenção da $\mathrm{Hb}$ dentro da faixa alvo é difícil, especialmente em períodos longos e a variabilidade ocorre mais frequentemente para valores mais elevados de $\mathrm{Hb}$.
\end{abstract}

UnITERMos: Anemia. Eritropoetina recombinante. Falência renal crônica.

\section{INTRODUÇÃO}

A anemia, um achado quase universal entre pacientes com doença renal crônica em fase terminal (DRC), está associada com aumento de mortalidade e piora da qualidade de vida ${ }^{1-3}$. Nos últimos 15 anos, diretrizes clínicas têm sido propostas em vários países com o objetivo de orientar o manejo da anemia em pacientes com DRC. Ao longo dos anos, houve alterações no manejo da anemia que levaram à contínua atualização destas diretrizes, sendo que os níveis de hemoglobina alvo se tornou matéria de contínuo debate ${ }^{4-6}$. Evidências atuais têm dado suporte à indicação de níveis de hemoglobina entre $11 \mathrm{~g} / \mathrm{dL}$ e $12,5 \mathrm{~g} / \mathrm{dL}$ como alvo para pacientes com DRC em diálise ${ }^{1,2}$. Mais recentemente, níveis elevados de hemoglobina foram associados com aumento do risco de complicações trombóticas, hipertensão e mortalidade ${ }^{7-9}$.

A manutenção dos níveis de hemoglobina dentro da faixa alvo se tornou um desafio importante dado a aparente relação

1. Doutor em Nefrologia pela Universidade Federal de São Paulo - UNIFESP, São Paulo, SP

2. Mestre em Nefrologia pela Universidade Federal de São Paulo - UNIFESP, São Paulo, SP

3. Médico Nefrologista pela Universidade Federal de São Paulo - UNIFESP, São Paulo, SP

4. Professor Livre-Docente da Disciplina de Nefrologia pela Universidade Federal de São Paulo - UNIFESP, São Paulo, SP

5. Professor Afiliado da Disciplina de Nefrologia da Universidade Federal de São Paulo - UNIFESP, São Paulo, SP

6. Professor Livre-Docente da Disciplina de Nefrologia da Universidade de São Paulo, Faculdade de Medicina São Paulo, SP

7. Médica Nefrologista do Instituto de Nefrologia e Diálise, Tabõao da Serra, SP

8. Médico Nefrologista do Instituto de Nefrologia e Diálise, São Paulo, SP

9. Médico Nefrologista do Instituto de Hemodiálise de Sorocaba, Sorocaba, SP

10. Gerente Médica Roche, Roche Brazil, São Paulo, SP

11. Gerente Médico Roche, Roche Brazil, São Paulo, SP

12. Professora Afiliada da Disciplina de Nefrologia da Universidade Federal de São Paulo - UNIFESP, São Paulo, SP 
de "curva em U" entre estes níveis e desfechos clínicos. Além disso, vários autores têm descrito o fenômeno da variabilidade da hemoglobina, que parece afetar mais de $90 \%$ dos pacientes em hemodiálise ${ }^{10-13}$. Como resultado, estima-se que somente $5 \%$ a $10 \%$ destes pacientes se mantêm dentro da faixa alvo de hemoglobina em períodos maiores ou iguais a seis meses ${ }^{12,14}$.

No Brasil, o manejo da anemia na DRC é caracterizado por dois aspectos importantes. Primeiro, desde os anos 90 os pacientes têm acesso gratuito à eritropoetina e terapia com ferro endovenoso. Segundo, a Sociedade Brasileira de Nefrologia publicou em 2000 um guia prático para orientar os profissionais de saúde no manejo da anemia e no uso destas medicações ${ }^{15}$. Nessa publicação foi sugerido como nível de hemoglobina alvos valores entre 11 e $12 \mathrm{~g} / \mathrm{dL}$. Nossa hipótese foi que estes dois aspectos poderiam facilitar o tratamento de anemia e reduzir a prevalência da variabilidade dos níveis de hemoglobina nos pacientes brasileiros em diálise. Portanto, o objetivo do estudo atual foi avaliar a proporção de pacientes cujos níveis de hemoglobina se mantiveram dentro da faixa alvo recomendada e investigar a variabilidade destes níveis ao longo do tempo em um grupo de pacientes em diálise.

\section{Métodos}

\section{Desenho do estudo e inclusão de pacientes}

Foi realizado um estudo retrospectivo conduzido em sete centros de diálise do Estado de São Paulo, SP, Brasil. Os pacientes incluídos no estudo foram aqueles que estavam em hemodiálise há pelo menos seis meses antes da coleta dos dados (janeiro 2004), recebendo tratamento com eritropoetina para correção de anemia por pelo menos três meses antes do período de estudo e que preenchiam alguns outros critérios de inclusão e nenhum de exclusão. Outros critérios de inclusão foram idade maior que 18 anos; média de hemoglobina entre $10,5 \mathrm{~g} / \mathrm{dL}$ e $12,5 \mathrm{~g} / \mathrm{dL}$ nos três meses anteriores ao período de inclusão. Nenhum paciente que iniciou diálise após janeiro de 2004 foi incluído no estudo. Os critérios de exclusão foram doença neoplásica maligna, exceto neoplasia cutâneo não-melanona, hemoglobinopatias, aplasia pura de célula branca, condição conhecida que levava à perda sanguínea, contagem de plaqueta maior que $500.000 / \mathrm{mm}^{3}$ no início do estudo e participação em outro estudo de intervenção nos três meses anteriores ao período de inclusão. A coleta de dados ocorreu entre janeiro de 2004 e dezembro de 2006. Os prontuários dos pacientes incluídos foram revisados sendo coletados dados demográficos, clínicos e parâmetros laboratoriais relativos aos três anos de avaliação. 0 protocolo do estudo foi aprovado pelo Comitê de Ética das instituições envolvidas e todos os pacientes incluídos forneceram um termo de consentimento livre e esclarecido.

\section{Avaliação da variabilidade da hemoglobina}

Foram registrados os valores da primeira hemoglobina mensal de cada paciente, obtida em cada instituição participante por metodologia padronizada. O nível de hemoglobina alvo considerado neste estudo foi aquele entre 10,5 g/dL e 12,5 g/dL. Durante o período do estudo foi obtido, mês a mês, a proporção de pacientes que apresentavam hemoglobina dentro deste alvo. Além disso, os pacientes forma divididos em seis categorias de acordo com o padrão de variabilidade de hemoglobina ao longo do tempo ${ }^{12}$ : (1) Baixo persistente, que compreendia pacientes com níveis de hemoglobina <10,5g/dL; (2) Constantemente dentro do alvo, que compreendia pacientes dentro da faixa alvo; (3) Alto persistente, pacientes com níveis $>12,5 \mathrm{~g} / \mathrm{dL}$; (4) Baixa amplitude de flutuação com hemoglobina baixa, que compreendia pacientes com hemoglobina baixa ou dentro do alvo; (5) Baixa amplitude de flutuação com hemoglobina alta, pacientes com hemoglobina alta ou dentro do alvo; e (6) Alta amplitude de flutuação, pacientes com hemoglobina baixa, alta ou dentro do alvo. Somente os pacientes com informações completas dos níveis de hemoglobina mensal foram incluídos nesta análise de variabilidade de hemoglobina.

\section{Análise estatística}

O tamanho da amostra foi estimado baseado na premissa que aproximadamente $30 \%$ dos pacientes com DRC em diálise tem níveis médios de hemoglobina fora da faixa alvo ${ }^{5}$. Dentro de um erro tipo I bicaudal de $5 \%$ e considerando uma taxa de perda de $20 \%$, a inclusão de 250 pacientes daria ao estudo um poder de $80 \%$ de encontrar uma proporção semelhante. Os dois parâmetros de interesse na análise atual foram a proporção de pacientes com níveis de hemoglobina dentro do alvo em cada mês durante o período de avaliação e a proporção de pacientes em cada categoria de flutuação de hemoglobina. Esta última proporção foi examinada durante períodos de tempo variando de seis a 36 meses. Todas as análises estatísticas foram realizadas com software SAS software (Statistical Analysis System, version 9.1.3).

\section{Resultados}

\section{Características dos pacientes}

As características clínicas e demográficas dos 249 pacientes incluídos neste estudo estão mostradas na Tabela 1 . Uma

Tabela 1 - Características basais dos pacientes $(\mathrm{N}=249)$

\begin{tabular}{lc}
\hline Parâmetros & Valores \\
\hline Idade, anos & $54(19$ a 86) \\
$\quad$ Mediana (variação) & $108(43,4)$ \\
Gênero, número (\%) & $141(56,6)$ \\
$\quad$ Feminino & \\
$\quad$ Masculino & $74(29,7)$ \\
Etiologia DRC, número (\%) & $57(22,9)$ \\
$\quad$ Hipertensão & $47(18,9)$ \\
$\quad$ Diabetes mellitus & $14(5,6)$ \\
$\quad$ Glomerulonefrite Crônica & $60(24,1)$ \\
Doença Renal Policística & \\
Outras ou não conhecida & $35(5$ a 175) \\
Tempo em hemodiálise, meses & \\
Mediana (variação) & $11,5(0,57)$ \\
Hemoglobina Basal, g/dL & \\
Média (desvio padrão) & $411,2(202,8)$ \\
Ferritina, ng/mL & \\
Média (desvio padrão) & $30(10,8)$ \\
Saturação de Transferrina \% & \\
Média (desvio padrão) &
\end{tabular}


proporção ligeiramente superior de pacientes do sexo masculino foram incluídos, a idade média foi de 54 anos, e o tempo médio em hemodiálise foi de 35 meses. A hipertensão arterial e a Diabetes mellitus contribuíram com aproximadamente

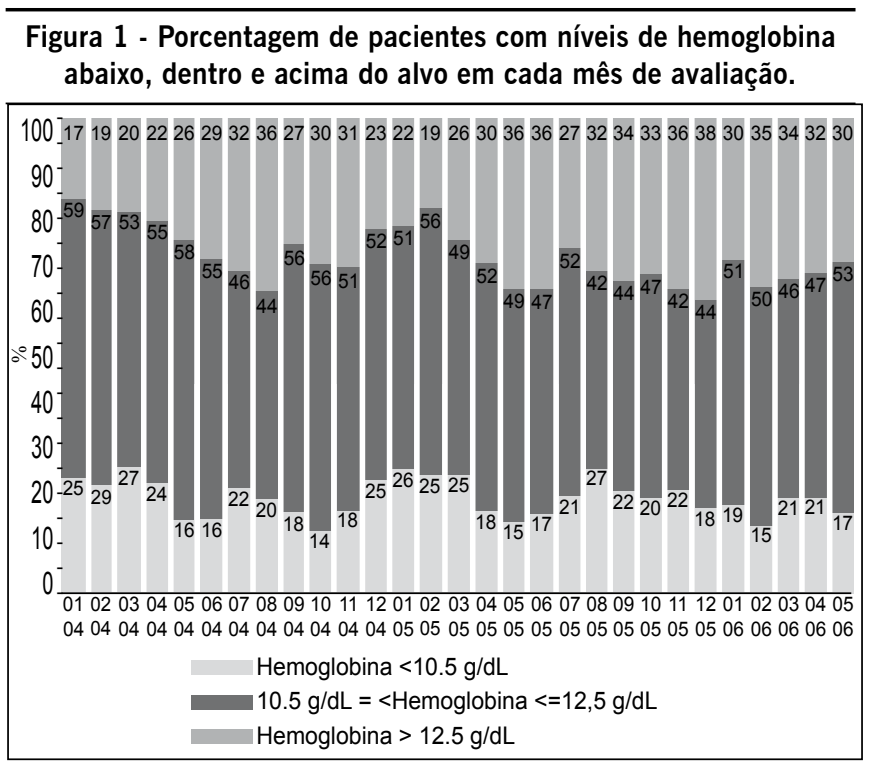

metade das causas de DRC nesta população. O valor médio de hemoglobina foi de $11,5 \mathrm{~g} / \mathrm{dL}$. Com exceção de um centro que incluiu nove pacientes, os outros incluíram de 34 a 49 pacientes cada um. Dos 249 pacientes incluídos, 143 (57,4\%) foram seguidos por todo o período do estudo. Dos pacientes que não completaram o seguimento, 54 pacientes $(21,7 \%)$ faleceram, $29(11,6 \%)$ foram submetidos a transplante renal e $23(9,2 \%)$ deixaram o estudo, pois trocaram de unidade de diálise ou de método dialítico.

\section{Níveis de hemoglobina alvo}

A proporção de pacientes mês a mês que estava dentro e fora do alvo de hemoglobina está mostrada na Figura 1. A proporção de pacientes com hemoglobina dentro do alvo em cada mês durante todo o período de estudo variou de $42 \%$ a $61 \%$, com uma média de $50 \%$. A proporção de pacientes com hemoglobina abaixo do alvo em cada mês variou de $17 \%$ a $27 \%$ (média de $20 \%$ ), e daqueles acima do alvo foi de $19 \%$ a $41 \%$ (média 30\%).

\section{Categorias de variabilidade de hemoglobina}

A proporção de pacientes em cada categoria de variabilidade de hemoglobina durante períodos de seis, 12, e 36 meses está mostrada na Tabela 2. A proporção de pacientes cujo nível de hemoglobina se manteve dentro do alvo variou de 5,9\% a $12,7 \%$, considerando períodos de seis meses, e entre $0,5 \%$ e

\begin{tabular}{|c|c|c|c|c|c|c|c|}
\hline \multirow{3}{*}{$\begin{array}{l}\text { Período de } \\
\text { Tempo }\end{array}$} & \multirow{3}{*}{$\mathrm{N}$} & \multicolumn{6}{|c|}{ Padrões de flutuação de hemoglobina } \\
\hline & & $\begin{array}{c}\text { Consistentemente } \\
\text { Baixo }\end{array}$ & $\begin{array}{c}\text { Consistentemente } \\
\text { No alvo }\end{array}$ & $\begin{array}{c}\text { Consistentemente } \\
\text { Alto }\end{array}$ & $\begin{array}{c}\text { Baixa } \\
\text { amplitude, } \\
\text { baixa HB }\end{array}$ & $\begin{array}{c}\text { Baixa } \\
\text { amplitude, } \\
\text { alto HB }\end{array}$ & $\begin{array}{c}\text { Alta } \\
\text { amplitude }\end{array}$ \\
\hline & & $\%$ & $\%$ & $\%$ & $\%$ & $\%$ & $\%$ \\
\hline \multicolumn{8}{|c|}{ Períodos de seis meses } \\
\hline $\begin{array}{l}07 / 04- \\
12 / 04\end{array}$ & 196 & 3,6 & 9,2 & 20,4 & 3,1 & $41, .3$ & 22,4 \\
\hline $\begin{array}{l}\text { 01/05- } \\
06 / 05\end{array}$ & 188 & 2,1 & 6,9 & 26,6 & 2,1 & 33,0 & 29,3 \\
\hline $\begin{array}{l}07 / 05- \\
12 / 05\end{array}$ & 172 & 2,9 & 6,4 & 26.2 & 7,6 & 35,5 & 21,5 \\
\hline \multicolumn{8}{|c|}{ Períodos de 12 meses } \\
\hline $\begin{array}{l}01 / 04- \\
12 / 04\end{array}$ & 188 & 0,5 & 0,5 & 19,7 & 0,5 & 26,1 & 52,7 \\
\hline $\begin{array}{l}01 / 05- \\
12 / 05\end{array}$ & 164 & 1,2 & 2,4 & 21,3 & 1,2 & 29,9 & 43,9 \\
\hline $\begin{array}{l}01 / 06- \\
12 / 06\end{array}$ & 130 & 0 & 2,3 & 9,2 & 0,8 & 29,2 & 58,5 \\
\hline \multicolumn{8}{|c|}{ Período de 36 meses } \\
\hline $\begin{array}{l}01 / 04- \\
12 / 06\end{array}$ & 108 & 0 & 0 & 2,8 & 0 & 8,3 & 88,9 \\
\hline
\end{tabular}


2,4\%, considerando períodos de 12 meses. Nenhum paciente manteve os níveis de hemoglobina dentro do alvo durante todo o período do estudo. Na análise de períodos de seis meses foi observada uma maior proporção de pacientes com flutuações de alta amplitude que de baixa amplitude. De forma semelhante houve uma maior proporção de pacientes com flutuação de baixa amplitude com hemoglobina alta (variação 29,4\% a 41,3\%) que com baixa amplitude e hemoglobina baixa (2,1\% a 7,6\%). De forma importante, $88,9 \%$ dos pacientes apresentaram flutuação de alta amplitude considerando todo o período de estudo. Foi realizada uma análise adicional considerando somente uma sub população de pacientes com níveis adequados de ferritina (pelo menos uma dosagem de ferritina $\geq 100 \mathrm{ng} / \mathrm{mL}$ nos períodos de seis meses e pelo menos duas dosagens de ferritina $\geq 100 \mathrm{ng} /$ $\mathrm{mL}$ para períodos de 12, 36 meses). Nesta análise, um padrão semelhante de variabilidade de hemoglobina foi observado (dados não mostrados).

\section{Discussão}

No estudo atual, dois resultados mais importantes foram encontrados. Primeiro, metade dos pacientes em diálise apresentava hemoglobina dentro do alvo nas avaliações mensais, e pacientes com hemoglobina alta foram mais frequentes que pacientes com baixa hemoglobina. Segundo, somente uma minoria de pacientes com DRC em hemodiálise apresentaram níveis de hemoglobina consistentemente dentro da faixa de $10,5 \mathrm{~g} / \mathrm{dL}$ a $12,5 \mathrm{~g} / \mathrm{dL}$, especialmente quando longo período de tempo foram analisados. Entre os pacientes foi observada uma variabilidade significante nos níveis de hemoglobina ao longo do tempo, e flutuações para valores mais altos foram mais frequentes que a tendência na direção oposta.

A definição da faixa alvo de hemoglobina compreende um limite de valores relativamente estreito. Estes níveis foram definidos considerando resultados obtidos em estudos observacionais que relataram que níveis de hemoglobina abaixo de 11 $\mathrm{g} / \mathrm{dL}$ estavam associados com maior prevalência de alterações cardiovasculares, piora da qualidade de vida e aumento das taxas de hospitalização e mortalidade. Por outro lado, estudos clínicos recentes mostraram que níveis elevados de hemoglobina aumentam o risco de complicações trombóticas, hipertensão não controlada e mortalidade ${ }^{7-9}$. Além disso, haveria possíveis efeitos tóxicos diretos resultantes do uso de doses não fisiológicas de eritropoetina ${ }^{16}$. As implicações destes achados não são totalmente conhecidas e outros estudos são necessários para caracterizar a ligação causal entre níveis de hemoglobina e eventos clínicos adversos. De fato, no estudo atual a média da porcentagem mensal de pacientes com hemoglobina acima de $12,5 \mathrm{~g} / \mathrm{dL}$ foi de $30 \%$ (variação $19 \%$ a $41 \%$ ).

Apesar do acesso universal a terapia com eritropoetina e ferro endovenoso no Brasil, somente uma minoria dos nossos pacientes tem níveis de hemoglobina persistentemente na faixa alvo, especialmente quando longos períodos são analisados. Os resultados do estudo atual estão de acordo com aqueles descritos por outros autores ${ }^{10-14}$. Em estudos retrospectivos realizados nos Estados Unidos em $2003^{12}$ e em 2004 ${ }^{17}$, aproximadamente $40 \%$ dos pacientes apresentaram flutuações de alta amplitude nos níveis de hemoglobina durante períodos de seis meses. No estudo atual, esta proporção vários de 21,5\% a 36,3\% na análise de período de seis meses. Entretanto, algumas diferenças entre os estudos americanos e o estudo atual têm que ser discutidas, já que um limite menor para a hemoglobina alvo foi adotado nos nossos pacientes $(10,5 \mathrm{~g} / \mathrm{dL}$, ao invés de $11 \mathrm{~g} / \mathrm{dL})$ e o fato de que os pacientes incluídos no estudo atual terem que apresentar uma média estável de hemoglobina nos três meses anteriores ao início do estudo. Em relação à variabilidade da hemoglobina, os pacientes no estudo atual apresentaram maior tendência que os pacientes dos estudos americanos, de estarem na categoria de níveis mais elevados de hemoglobina e menor tendência de estarem no grupo com níveis mais baixos de hemoglobina. Estes achados poderiam refletir o acesso universal ou um uso mais agressivo de terapia com eritropoetina no grupo de pacientes avaliados.

Em um estudo retrospectivo de 1996 do Fresenius Medical Care a variabilidade de hemoglobina foi associada com aumento de mortalidade ${ }^{18}$. De forma importante, Ebben et al. mostraram que flutuações de alta amplitude foi um importante fator de risco para hospitalização em pacientes com DRC em hemodiálise, sendo apenas menos importante que a categoria de hemoglobina persistentemente baixa ${ }^{12}$. 0 mesmo grupo mostrou, posteriormente, um aumento no risco de óbito entre pacientes com flutuação de alta amplitude em comparação com pacientes cujos níveis de hemoglobina se mantiveram dentro da faixa alvo ${ }^{17}$. Se a flutuação de alta amplitude está ligada de forma causal aos eventos adversos ou é apenas um marcador de outras situações associadas à variabilidade de hemoglobina é também uma questão que necessita maiores investigações.

O estudo atual apresenta duas principais limitações: 0 número relativamente pequeno de pacientes avaliados e a natureza retrospectiva do estudo. O número de pacientes neste estudo é menor que os estudos prévios que avaliaram a variabilidade de hemoglobina em pacientes com DRC em hemodiálise 10,12,14,19,20. Entretanto, os pacientes incluídos no estudo atual representam uma população relativamente homogênia e poderiam refletir os pacientes brasileiros com DRC em hemodiálise. Em relação à natureza retrospectiva deste estudo, este tipo de desenho pode interferir com a avaliação dos fatores que influenciam os padrões de variabilidade de hemoglobina. Entretanto, a análise destes fatores não foi o objetivo do estudo atual. Além disso acreditamos que os resultados descritos refletiram a conduta real dos centros envolvidos no estudo já que a prescrição de eritropoetina era totalmente dependente da decisão da equipe medida de cada unidade de diálise.

\section{Conclusão}

Em conclusão, apesar do acesso universal à terapia com eritropoetina e ferro endovenoso no Brasil e a disponibilidade de guias clínicos para o manejo da anemia, a manutenção dos níveis de hemoglobina dentro de uma faixa alvo é difícil, já que a maioria dos pacientes experimentou flutuação de alta amplitude ao longo do tempo. Além disso, a proporção de pacientes que não atingiram o alvo de hemoglobina aumentou quando períodos de tempo maiores formam analisados. Finalmente, a variabilidade pareceu ocorrer mais frequentemente para valores mais elevados de hemoglobina que para níveis mais baixos. Estudos adicionais são necessários para determinar as consequências destes achados e complementar as diretrizes clínicas para o manejo da anemia em pacientes com DRC em diálise. 


\section{SUPORTE FinANCEIRO:}

A empresa Roche forneceu recursos para este trabalho. Os investigadores foram responsáveis pelo desenho, condução e análise deste estudo. Não há restrições para a publicação. Dra. Canziani refere que recebe recursos da Roche pelo trabalho de consultoria.

\section{Conflito de interesse: não há}

\section{SUMMARY}

\section{Hemoglobin levels in hemodialysis patients treated with EPOETIN: A BRAZILIAN EXPERIENCE}

OBJECTIVE. Correction of anemia using epoetin decreases morbidity and increases survival and quality of life in endstage renal disease. Maintaining hemoglobin levels within the range proposed by guidelines has become a major challenge, with hemoglobin cycling affecting more than $90 \%$ of patients undergoing hemodialysis. The variability of hemoglobin levels over time was assessed in our patients.

METHODS. Data were retrospectively collected on 249 patients undergoing hemodialysis over a 3-year period at seven centers in Brazil. Hemoglobin was measured at least monthly, and target levels were those between $10.5 \mathrm{~g} / \mathrm{dL}$ and $12.5 \mathrm{~g} /$ $d L$. Patients were grouped into six categories of variability consistently low $(<10.5 \mathrm{~g} / \mathrm{dL})$, consistently target range $(10.5$ to $12.5 \mathrm{~g} / \mathrm{dL}$ ), consistently high (>12.5g/dL), low amplitude fluctuation with low hemoglobin levels, low amplitude fluctuation with high hemoglobin levels and high amplitude fluctuation. None of the patients maintained stable hemoglobin levels for the entire 36-month period.

RESULTS. The mean monthly proportion of patients that had hemoglobin levels within the target range was 50\% (range, $42 \%$ to $61 \%$ ). Mean levels above the target (30\%) were more frequent than those below it (20\%). During 6, 12, and 36 months, proportions of patients with consistently low levels of hemoglobin decreased from $3.6 \%$ to $0 \%$, from $31.7 \%$ to $2.8 \%$ for those with consistently high, from $7.6 \%$ to $0 \%$ for those with low amplitude fluctuation with low hemog/obin levels and from $41.3 \%$ to $8.3 \%$ for those with low amplitude fluctuation with high hemog/obin levels. However, the proportions of patients with high amplitude fluctuation increased from $21.5 \%$ to $88.9 \%$.

CONCLUSION. Maintaining hemoglobin levels within the target range is difficult, especially for longer periods of time. Missing the target seems more often due to levels above it, but highamplitude fluctuations eventually occur in the majority of patients. [Rev Assoc Med Bras 2010; 56(2): 209-13]

KEY wORDS: Anemia. Erythropoietin. Recombinant. Kidney failure. Chronic.

\section{REFERÊNCIAS}

1. Locatelli F, Pisoni RL, Combe C, Bommer J, Andreucci VE, Piera L, et al. Anaemia in haemodialysis patients of five European countries: association with morbidity and mortality in the Dialysis Outcomes and Practice Patterns Study (DOPPS). Nephrol Dial Transplant. 2004;19:121-32.

2. Robinson BM, Joffe MM, Berns JS, Pisoni RL, Port FK, Feldman HI. Anemia and mortality in hemodialysis patients: accounting for morbidity and treatment variables updated over time. Kidney Int. 2005;68:2323-30.

3. Valderrabano F. Quality of life benefits of early anaemia treatment. Nephrol Dial Transplant. 2000;15(Suppl 3):23-8.

4. Locatelli F, Aljama P, Barany P, Canaud B, Carrera F, Eckardt KU, et al. Revised European best practice guidelines for the management of anaemia in patients with chronic renal failure. Nephrol Dial Transplant. 2004;19(Suppl 2):ii 1-47.

5. KDOQI Clinical Practice Guidelines and Clinical Practice Recommendations for Anemia in Chronic Kidney Disease. Am J Kidney Dis. 2006;47:S11-145.

6. Manns BJ, White CT, Madore F, Moist LM, Klarenbach SW, Barrett BJ, et al. Introduction to the Canadian Society of Nephrology clinical practice guidelines for the management of anemia associated with chronic kidney disease. Kidney Int Suppl. 2008:S1-3.

7. Strippoli GF, Craig JC, Manno C, Schena FP. Hemoglobin targets for the anemia of chronic kidney disease: a meta-analysis of randomized, controlled trials. J Am Soc Nephrol. 2004;15:3154-65.

8. Singh AK, Szczech L, Tang KL, Barnhart H, Sapp S, Wolfson M, Reddan D. Correction of anemia with epoetin alfa chronic kidney disease. N Engl J Med. 2006;355:2085-98.

9. Phrommintikul A, Haas SJ, Elsik M, Krum H. Mortality and target haemoglobin concentrations in anaemic patients with chronic kidney disease treated with erythropoietin: a meta-analysis. Lancet. 2007;369:381-8.

10. Berns JS, Elzein H, Lynn RI, Fishbane S, Meisels IS, Deoreo PB. Hemoglobin variability in epoetin-treated hemodialysis patients. Kidney Int. 2003;64:1514-21

11. Fishbane S, Berns JS. Hemoglobin cycling in hemodialysis patients treated with recombinant human erythropoietin. Kidney Int. 2005;68:1337-43.

12. Ebben JP, Gilbertson DT, Foley RN, Collins AJ. Hemoglobin level variability: associations with comorbidity, intercurrent events, and hospitalizations. Clin J Am Soc Nephrol. 2006;1:1205-10.

13. Mendonça TA, Oliveira RA, Andrade Júnior MP, Bastos KA. Variabilidade da hemoglobina e hospitalização em pacientes com doença renal crônica em programa dialítico em uso de epoetina alfa. J Bras Nefrol. 2008;30:272-9

14. Lacson E Jr, Ofsthun N, Lazarus JM. Effect of variability in anemia management on hemoglobin outcomes in ESRD. Am J Kidney Dis. 2003;41:111-24.

15. Abensur H. Alves MR. Diretrizes da Sociedade Brasileira de Nefrologia para a condução da anemia na insuficiência renal crônica. J Bras Nefrol. 2000;22(Supl 5):1-3.

16. Rosner $\mathrm{MH}$, Bolton WK. The mortality risk associated with higher hemoglobin: is the therapy to blame? Kidney Int. 2008;74:695-7.

17. Gilbertson DT, Ebben JP, Foley RN, Weinhandl ED, Bradbury BD, Collins AJ. Hemoglobin level variability: associations with mortality. Clin J Am Soc Nephrol. 2008;3:133-8.

18. Brunelli SM, Joffe MM, Israni RK, Yang W, Fishbane S, Berns JS, et al. Historyadjusted marginal structural analysis of the association between hemoglobin variability and mortality among chronic hemodialysis patients. Clin J Am Soc Nephrol. 2008;3:777-82.

19. Li S, Foley RN, Gilbertson DT, Liu J, Collins AJ. Clinical factors associated with achieving $\mathrm{K} / \mathrm{DOQ}$ hemoglobin targets in hemodialysis patients. Int Urol Nephrol. 2003;35:399-405.

20. Collins AJ, Brenner RM, Ofman JJ, Chi EM, Stuccio-White N, Krishnan M, et al. Epoetin alfa use in patients with ESRD: an analysis of recent US prescribing patterns and hemoglobin outcomes. Am J Kidney Dis. 2005;46:481-8.
Artigo recebido: 16/6/09

Aceito para publicação: 25/10/09 